\title{
APP and its secreted fragment SAPP in SH-SY5Y neuronal-like migration
}

\author{
Pinho, A.C.*, Dias, R.*, Cerqueira, A.R.*, da Cruz e Silva, O.A.B.*, Vieira, S.I.*\# \\ *Neuroscience Lab., Center for Cell Biology, Health Sciences Dept. and Biology Dept., University of \\ Aveiro, Campus de Santiago, 3810-193 Aveiro, PORTUGAL \\ Email: sivieira@ua.pt
}

During adult neurogenesis, neural stem cells produce neural progenitors that migrate toward their final location, in a process highly regulated via signaling cues and cellular sensors[1]. The Alzheimer's Amyloid Precursor Protein (APP) is an ubiquitously expressed transmembranar protein that has been implicated in cell migration, together with its secreted proteolytic fragment, sAPP [2]. Although the latter was observed to be motogenic for keratinocytes, via inducing cell polarity and by increasing both the migrating keratinocytes population and their migration velocity[3], less is known of its role in neuronal migration.

In order to study the paracrine role of sAPP in neuronal-like cells migration, we performed Scratch Wound Healing (SWH) assays in SH-SY5Y neuroblastoma cells. Migration of non-transfected (NTf) cells was monitored in different backgrounds: a) in the vicinity of GFP (Green Fluorescent Protein) transiently transfected cells (EGFP-N1); b) in the vicinity of cells transfected with wild-type APP cDNA constructs fused to GFP [4] (Wt APP-GFP); and c) in the vicinity of GFP transfected cells in an sAPP-enriched medium (EGFP-N1+sAPP). The "healing" of the "wound gap" by cell migration was monitored by imaging both wound edges at every hour, during $8 \mathrm{~h}(\mathrm{t} 0-\mathrm{t} 8)$, and in the following day (t24), as depicted in phase contrast $(\mathrm{PhC})$ microphotographs of Fig.1A. Cell migration efficiency was evaluated by determination of the following parameters: 1) the migrating cells score (number of NTf cells observed at the wound area with time); 2) their fold increase over time (reflecting the recruitment of new cells to the migratory process), and 3 ) the final distance migrated by the NTf's leading edge (distance measured in a direct line, from the t0 edge of the wound - red line in Fig.1 A - to the average line passing by ten random leading edge cells at 24h).

Results show that the presence of higher sAPP levels that gradually accumulated in the media via Wt APPGFP proteolysis (Fig.1B, 'APP-GFP') did not increase but rather slightly decreased the absolute number of initial NTf migrating cells (Fig.2A). Nonetheless, the final number of these migratory cells equaled the control EGFP-N1 condition (Fig.2A, 24h), since cells in the Wt APP-GFP condition suffered a delayed but enhanced recruitment for migration from $4 \mathrm{~h}$ on (Fig.2B, arrow), as demonstrated by its highest fold increases thereafter (Fig.2B, 4h on). Intriguingly, NTf cells in the EGFP-N1+sAPP condition generally presented the lowest number of migrating cells (Fig.2A), despite its intermediate time pattern of cell recruitment (Fig.2B). These data suggest that sAPP may be responsible for the late enhanced cell recruitment to migration seen in Wt APP-GFP. However, at higher doses (EGFP-N1+sAPP) it decreases the number of cells migrating into the wound area, what may result from sAPP-induced negative feedbacks and/or desensitization mechanisms. Since in this condition there is no real sAPP gradient (it is homogenously distributed throughout cells media), this may also add a negative effect on directed cells migration. In this case cells would be activated to migrate but in a random manner and not towards the center of the gap. Findings that in the EGFPN1+sAPP condition the NTf cells leading edge migrated shorter distances over time (data not shown), support the 'loss of migration directionality' hypothesis, with this being further pursued by us. With this work we conclude that sAPP may have a paracrine role recruiting neuronal cells for migration, but possibly in a dose- and gradient-dependent manner.

Acknowledgements: Supported by CBC/UA; FCT/COMPETE/QREN/EU (PTDC/SAUNMC/111980/2009, SFRH/BD/90996/2012, PEst-OE/SAU/UI0482/2013). 


\section{References:}

[1] Cayre M. et al., Prog Neurobiol, 88, 41-63,2009.

[2] Zhou Z. et al., Cell Adhesion \& Migration, 5, 280-292, 2011.

[3] Kirfel G. et al., European journal of cell biology, 81, 664-676, 2002.

[4] Vieira et al., Molecular and Cellular Biochemistry, 328, 145-154, 2009.

A.

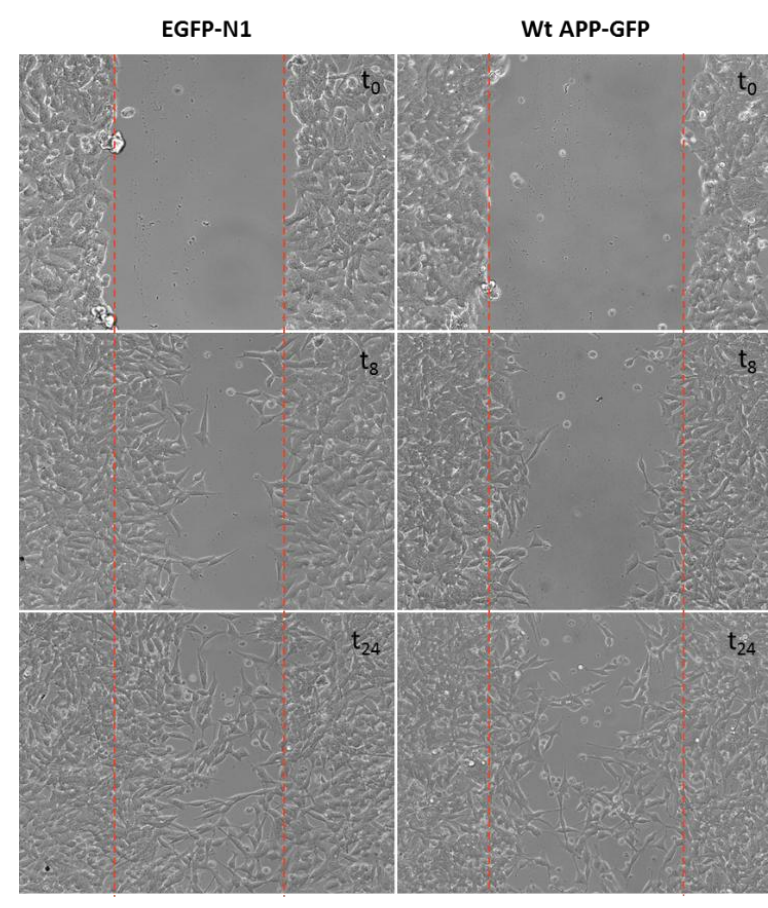

A.

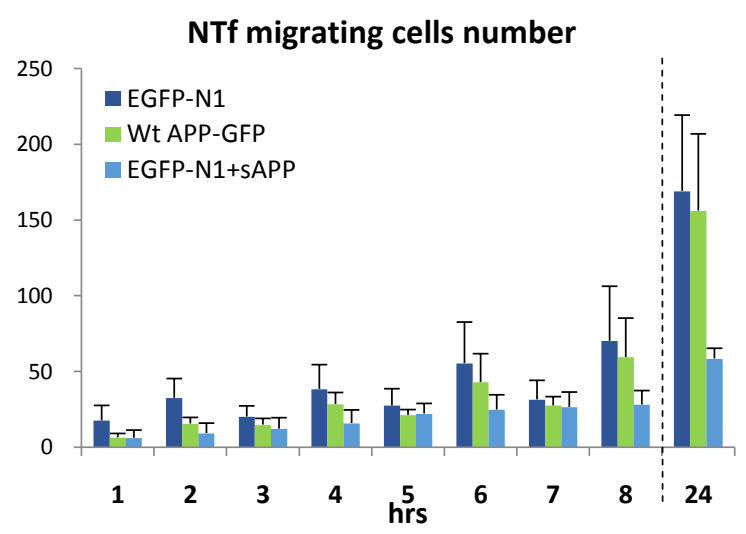

\section{B. SAPP in media:}

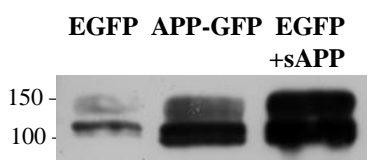

Figure $\quad 1 . \quad$ A) Representative $\mathrm{PhC}$ microphotographs of a SWH assay in SHSY5Y cells in EGFPN1, Wt APPGFP and EGFP-N1+sAPP expression backgrounds. Photographs were taken immediately after scratching ( $\mathrm{t} 0)$, or 8h (t8) and 24h later (t24). Red parallel lines delimit the edges

Figure 2. Number of non-transfected migrating cells entering the wound area at the indicated time points in EGFP-N1, Wt APP-GFP, and EGFP-N1+sAPP expression backgrounds. A. Absolute numbers. B. Fold increases over $\mathrm{t} 2(2 \mathrm{~h})$. The analysis was performed in microphotographs taken every hour until $8 \mathrm{~h}$ of migration and upon $24 \mathrm{~h}$ of migration. Data are presented as mean \pm standard error of the mean of 3 to 4 independent experiments. NTf, non-transfected cells. 